\author{
R. MATTHEY, \\ S. SCHILT ${ }^{2}$ \\ D. WERNER ${ }^{2}$ \\ C. AFFOLDERBACH ${ }^{1}$ \\ L. THÉVENAZ ${ }^{2}$ \\ G. MILETI ${ }^{1}$
}

\section{Diode laser frequency stabilisation for water-vapour differential absorption sensing}

\footnotetext{
${ }^{1}$ Observatoire cantonal de Neuchâtel, Rue de l'Observatoire 58, 2000 Neuchâtel, Switzerland

${ }^{2}$ Ecole Polytechnique Fédérale de Lausanne (EPFL), Nanophotonics and Metrology Laboratory, 1015 Lausanne, Switzerland
}

\section{Received: 14 April 2006/Revised version: 24 May 2006} C) Springer-Verlag 2006

\begin{abstract}
We describe a low-power continuous-wave laser system for water-vapour sensing applications in the 935-nm region. The system is based on extended-cavity diode lasers and distributed-feedback lasers and delivers four single-mode frequency-stabilised optical signals. Three lasers are locked to three water-vapour absorption lines of different strengths, whereas the fourth lies outside any absorption line. On-line stabilisation is performed by wavelength-modulation spectroscopy using compact water-vapour reference cells. An offset-locking technique implemented around an electrical filter is applied for the stabilisation of the off-line slave laser to an on-line master laser at a frequency detuning of $18.8 \mathrm{GHz}$. Stabilities in the order of $15 \mathrm{MHz}$ over one day were observed for the strongest lines, at the detection limit of the measurement instrumentation. The developed techniques and schemes can be applied to other wavelength ranges and molecular species. Differential absorption lidar instrumentation can in particular benefit from such a system when the stabilised lasers serve as injection seeders to pulsed power oscillators.
\end{abstract}

PACS 42.62.Fi; 42.72.-g; 42.68.Wt

\section{$1 \quad$ Introduction}

Water vapour is a key component in the atmosphere. As part of the global water cycle it strongly influences the surface/atmosphere interaction processes. Its phase transitions determine the transport and redistribution of energy around the globe, affecting the weather. It plays a part in many atmospheric chemistry processes and, as a major greenhouse gas, plays a central role in the climate. Improved water-vapour measurements are needed in numerous disciplines and scientific issues [1]. For meteorology, weather forecasting, climatology as well as atmospheric process studies, it is important to accurately monitor both the temporally and spatially variable water-vapour concentration, not only through point or integrated column measurements, but also with a high spatial and temporal resolution.

The water-vapour differential absorption lidar (DIAL) technique provides an independent and direct manner to retrieve the atmospheric humidity profile in a continuous way,

Fax: +41-32-722-04-20, E-mail: renaud.matthey@ne.ch with a high temporal and spatial resolution [2-4]. It is based on the comparison of the lidar signals at two wavelengths, one coinciding with a water-vapour absorption line of adequate strength and the second sufficiently far away from any line not to undergo substantial absorption. The quality of the data obtained from ground-based [5,6] and airborne [7-10] instruments in terms of vertical resolution, precision and accuracy indicates the feasibility of a space-borne instrument [11], from which major advances in humidity profiling with global coverage are expected [12]. While ground-based water-vapour DIALs operate at two wavelengths only (one on-line and the other off-line), space-borne instruments may operate at a set of different on-line wavelengths corresponding to absorption lines of different strengths, thus providing different penetration depths in the atmosphere. Strongly absorbing water-vapour lines are used for higher altitudes, whereas weakly absorbing lines serve for lower altitudes, which show high water-vapour concentrations. The simultaneous use of four different wavelengths (three on-line and one off-line) enables measurement of $\mathrm{H}_{2} \mathrm{O}$ profiles across the entire altitude ranging from the lower stratosphere to the boundary layer. The specific wavelengths must be selected depending on the atmospheric process under study, the desired altitude resolution and coverage and the required measurement precision [13].

For reliable water-vapour DIAL measurements, high spectral purity as well as short- and long-term stabilities (and accuracies) of the lidar operating wavelengths are of crucial importance [4]. At the same time, the transmitter output power must be high enough to ensure a sufficiently large signal-to-noise ratio of the atmospheric measurements. An efficient way to achieve these requirements is to make use of the injection-seeding technique; a single-mode, narrow-linewidth, low-power, continuous-wave (cw) laser that fulfils the necessary stability conditions seeds a power oscillator, imprinting its spectral properties. Seeding a Ti:sapphire laser in the $820-\mathrm{nm}$ region $[14,15]$ and an optical parametric oscillator operating around $935 \mathrm{~nm}$ [16] are examples of such a technique implemented in water-vapour DIAL instruments.

In this paper, we report the development and evaluation of a demonstrator for a laser system frequency stabilised to the water-vapour absorption spectrum, which could serve as a master laser to a power oscillator for a possible fourwavelength water-vapour space-borne DIAL instrument. Our system simultaneously delivers four reference wavelengths, 
three on-line coinciding with $\mathrm{H}_{2} \mathrm{O}$ absorption lines of different strengths and the fourth being locked off-line. To our knowledge, the implemented off-line laser locking scheme allows frequency stabilisation to a precise wavelength at a level not previously reached in a DIAL system.

\section{Overview of the system}

The 935-nm wavelength region offers water-vapour absorption lines with appropriate strengths for high-quality DIAL measurements in the troposphere and the lower stratosphere [8]. The most intense lines in this wavelength range are one order of magnitude stronger than those in the 730- or 820-nm absorption bands that are usually used in tropospheric water DIAL systems. A critical constraint in the selection of suitable water absorption lines is that the lower-state energy of the molecular transitions must be low enough to minimise the temperature dependence of the absorption over the target altitude range (see [4] and [13] and included references for a detailed discussion about the line-selection criteria). Figure 1 depicts the water-vapour spectrum around $935 \mathrm{~nm}$. The centres of the lines referred to as strong $(\mathrm{S})$, medium $(\mathrm{M})$ and weak (W) with respect to their relative strengths are, together with the off-line wavelength $(\mathrm{O})$, the frequency references considered in this work. Their parameters are listed in Table 1 according to the water-vapour European Space Agency (ESA) database [17]. For a downward-looking instrument embarked onboard an aircraft or on a satellite, the S, M and W lines cover approximately the lower stratosphere, free troposphere and boundary layer, respectively.

The basic principle of the developed multi-wavelength reference source is schematically depicted in Fig. 2. Four independent, $\mathrm{cw}$ diode lasers are stabilised to the corresponding frequencies (at the centres of the $\mathrm{S}, \mathrm{M}$ or $\mathrm{W}$ water-vapour lines and off-line wavelength, respectively) using a separate frequency reference and a dedicated stabilisation loop for each laser. A part of each laser output power is split off and constitutes the output of the system for further injection seeding. Two different types of lasers have been used in the system in order to demonstrate their respective suitability for such a multi-wavelength reference system: (i) laboratory-developed extended-cavity diode lasers (ECDLs) that involve optical feedback from a mechanically moving diffraction grating and (ii) prototypes of distributed-feedback (DFB) laser diodes.

The laser stabilisation loops serve to reduce or even remove the laser frequency fluctuations due to environmental (temperature) or pumping (laser current variation) perturbations. For stabilisation of the on-line wavelengths (S, M and

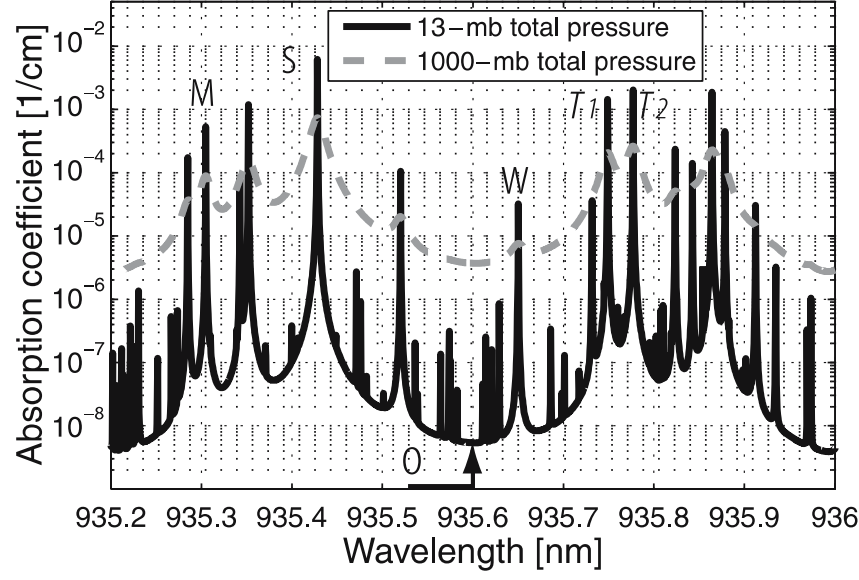

FIGURE 1 Water-vapour absorption spectrum in the 935-nm region calculated at ambient temperature $(296 \mathrm{~K})$ for 13 -mbar of pure water vapour (straight line) and for 13-mbar of $\mathrm{H}_{2} \mathrm{O}$ at 1000-mbar total pressure environment (dashed line). In addition to the $\mathrm{S}, \mathrm{M}, \mathrm{W}$ and $\mathrm{O}$ lines, $\mathrm{T}_{1}$ and $\mathrm{T}_{2}$ are lines used for tests

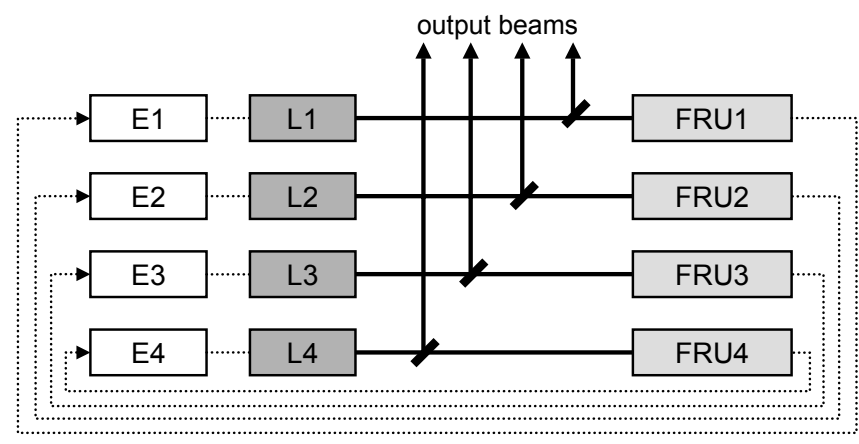

FIGURE 2 Schematic diagram of the multi-wavelength reference system. L: laser, FRU: frequency reference unit, E: electronics module for laser control and feedback loop for frequency locking

W), direct locking of the lasers to water-vapour absorption lines serving as frequency references is implemented. In contrast, an offset-locking scheme is used to stabilise the off-line laser to a well-defined frequency detuning relative to a reference absorption line. In our system the offset-locking scheme creates an artificial reference line at the desired off-line wavelength. Thus, the electronics for direct locking can be used for all four lasers, which greatly simplifies the overall design.

The system package (without the electronics modules) is shown in Fig. 3. It is mounted on an optical breadboard of dimensions $750 \times 530 \times 150 \mathrm{~mm}^{3}$. The system is realised in free-space optics since several components such as laser diodes and suitable reference cells were not available in a fibre-pigtailed version (apart from being more compact, an

\begin{tabular}{lcccccc}
\hline Line & $v\left[\mathrm{~cm}^{-1}\right]$ & $\lambda_{\text {air }}[\mathrm{nm}]$ & $S\left[\mathrm{~cm}^{-1} /\left(\mathrm{mol} \mathrm{cm}^{-2}\right)\right]$ & $E^{\prime \prime}\left[\mathrm{cm}^{-1}\right]$ & $g\left[\mathrm{~cm}^{-1}\right]$ & $g_{\mathrm{s}}\left[\mathrm{cm}^{-1}\right]$ \\
\hline Strong & 10687.363 & 935.4283 & $6.83 \times 10^{-22}$ & 136.759 & 0.0945 & 0.4855 \\
Medium & 10688.7726 & 935.3049 & $5.78 \times 10^{-23}$ & 488.114 & 0.0736 & 0.3783 \\
Weak & 10684.8292 & 935.6501 & $3.58 \times 10^{-24}$ & 382.521 & 0.1009 & 0.68 \\
Off-line & 10685.4016 & 935.600 & - & - & - & - \\
\hline
\end{tabular}

TABLE 1 Parameters of the individual lines used for the stabilisation of the four lasers. $v$ is the transition wavenumber, $\lambda_{\text {air }}$ the wavelength in air (calculated with the air refraction index $n=1.0002739$ ), $S$ the line strength, $E^{\prime \prime}$ the lower-state energy, $g$ and $g_{\mathrm{s}}$ the air- and self-broadening coefficients (HWHM) and $n$ the temperature-dependence coefficient 


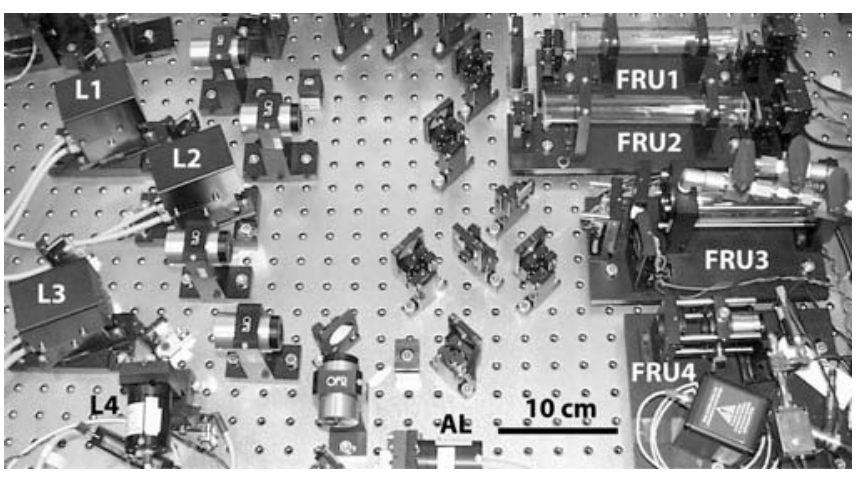

FIGURE 3 Photograph of the realised multi-wavelength reference system. Left-hand side: the four laser units (L1 to L4). Right-hand side: the four frequency reference units (FRU1 to FRU4 for lines S, M, W and O, respectively, from top to bottom). Middle part: transfer optics (optical isolators and beam splitters) and an additional fifth laser (AL) for test and characterisation purposes

optical fibre configuration would be less prone to optical misalignment). Each of the four lasers is protected against optical feedback by an optical isolator. An additional laser that can replace any other laser (by insertion of a mirror at a proper location) was introduced into the system for tests and system characterisation. Laboratory laser electronics completes the system and a computer-controlled monitoring system records the system operation parameters during long-term evaluation.

\section{Laser sources}

The ECDLs and DFB lasers implemented in the system were subject to in-depth characterisation. Each laser type offers some advantages but also has some drawbacks compared to the other for implementation in the reference system. The ECDLs, designed from a stabilised laser head that was previously developed for $\mathrm{Rb}$ atomic clocks [18], can be tuned over more than $35 \mathrm{~nm}$ with a mode-hop-free tuning range of $10 \mathrm{GHz}$. On the other hand, the DFB lasers are intrinsically single mode with a large mode-hop-free tuning range of several nanometres, but the tuning range around their operation central wavelength is small in comparison to that of an ECDL. As they contain no moving part, they are more compact and stable than the ECDLs; they are well suited for airborne or space-borne applications. Presently, such DFB lasers can deliver up to $10 \mathrm{~mW}$ of optical power,

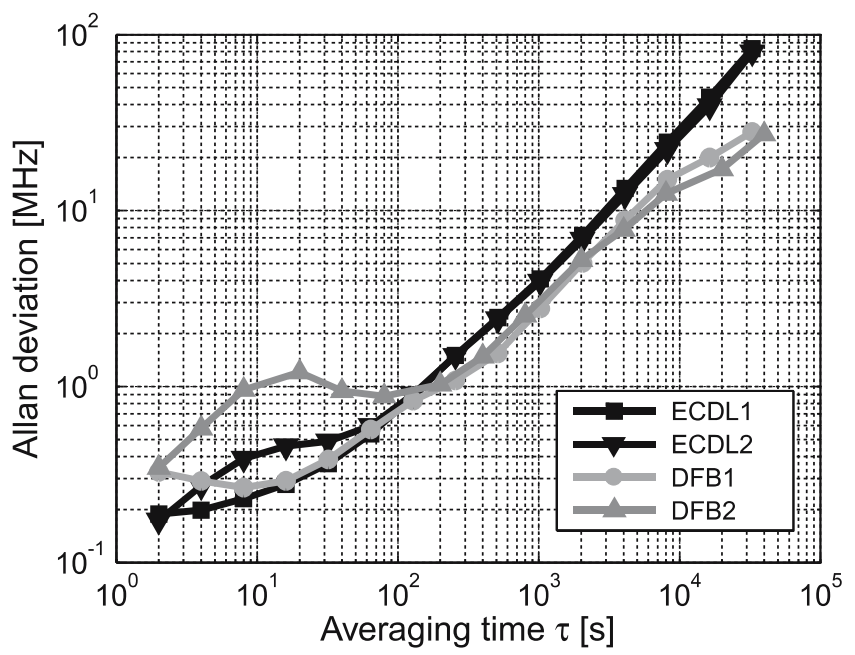

FIGURE 4 Frequency stability of the free-running lasers in terms of the Allan deviation. The bump features observed between 4 and $100 \mathrm{~s}$ stemmed from interference fringes in the optical setup

which is likely not sufficient for frequency stabilisation and injection seeding purposes. Instead, the output power of the ECDLs is about three times higher. The compact $(85 \times 82 \times$ $45 \mathrm{~mm}^{3}$ ) ECDLs demonstrated narrower line width than the DFB lasers, $300 \mathrm{kHz}$ and $2.7 \mathrm{MHz}$, respectively, as determined by heterodyne detection. Yet, neither of these values should be a limiting factor for injection seeding of a power oscillator. The passive stability represents another important laser parameter. High passive stability relaxes the requirement on the stabilisation loop. Using frequency discriminators, we measured that all free-running lasers were stable to better than $5 \mathrm{MHz}$ over a time interval of $1000 \mathrm{~s}$ (see Fig. 4), once they were placed in a protective box, where the temperature fluctuated by less than $0.1{ }^{\circ} \mathrm{C}$ over the same time interval. The main laser characteristics are listed in Table 2 together with the requirements for their deployment in a space-borne DIAL.

The frequency of our ECDLs can be modulated in two different ways for the generation of the error signal for laser stabilisation: through injection current modulation and through a modulation of the piezo-electrical transducer (PZT) controlling the length of the external cavity. Only current modulation can be applied to DFB lasers. Current modulation has the advantage of high bandwidth (up to $\mathrm{GHz}$ ), but has the draw-

\begin{tabular}{lccc}
\hline Parameter & Requirement & ECDL & DFB \\
\hline Wavelength [nm] & 935.4 & 935.4 & 935.4 \\
Threshold current [mA] & - & 30 & 9 \\
Current (operational) [mA] & - & 100 & $30-35$ \\
Optical power [mW] & $20-30$ & $30-40$ & 10 \\
Laser diode temperature (operational) [ $\left.{ }^{\circ} \mathrm{C}\right]$ & - & 20 & $27-30$ \\
Slope efficiency [mW/mA] & - & -0.4 & 0.5 \\
Tuning efficiency (ECDL: PZT; DFB: current) & - & $-400 \mathrm{MHz} / \mathrm{V}$ & $-2.6 \mathrm{GHz} / \mathrm{mA}$ \\
intensity modulation-frequency modulation (IM-FM) & & - & $\sim-20$ \\
$\quad$ phase shift (DFB only at 50 kHz) [deg] & - & 0.3 & 2.7 \\
Line width (1 ms) [MHz] & $<50$ & 0.3 & 1 \\
Free-running frequency stability (14 s) [MHz] & $<500$ & 200 & 100 \\
Free-running frequency drift (24 h) [MHz] & - & $<4 \times 10^{-3}$ & $<2 \times 10^{-3}$ \\
Relative optical power stability (14 s) [\%] & - & 0.12 & 0.04 \\
Relative optical power stability (3 h) [\%] & & & 5 \\
\hline
\end{tabular}

TABLE 2 Overview of the laser performances. Requirements refer to values retained as objectives for space applications (airborne and ground-based applications are less demanding). Similar performances were obtained between different lasers of the same type. Stabilities and drifts are expressed in term of the Allan deviation 
back of inducing a modulation of the laser output power. On the other hand, the laser output power may be kept almost constant when the modulation signal is applied to the PZT element in an ECDL. In this case, the modulation frequency is typically limited to a few $\mathrm{kHz}$.

\section{$4 \quad$ Stabilisation schemes \\ 4.1 On-line locking}

Laser stabilisation to water-vapour lines has been achieved by wavelength-modulation spectroscopy (WMS) [19], a technique in which the wavelength of a laser is dithered around the considered absorption line and which generates derivative-like signals at the successive harmonics of the modulation frequency. For pure laser wavelength modulation (WM), the odd derivatives cross zero at the centre of the absorption line and vary linearly around this point, so that they can act as an error signal in a servo loop. The application of WMS to the stabilisation of a laser source on water-vapour lines in the near infrared has been demonstrated [20-23], but generally only for a single stabilised laser. The same basic principle was applied in this work for the stabilisation of the three water lines, but its practical implementation differed slightly due to the different strengths of the absorption lines and to the influence of the residual intensity modulation (IM) of the laser.

WM is often accompanied by an undesired residual IM, in particular for laser modulation through the injection current; this effect can also occur to a lesser extent when tuning the wavelength of an ECDL with its grating, due to possible grating-angle-dependent reflectivity. The critical consequence, especially for small absorbance, is the appearance of a background offset in the first harmonic $(1 f)$ signal and a resulting shift of its zero-crossing point from the line centre, which leads to laser locking to an inaccurate frequency. Demodulation at the third harmonic $(3 f)$ is a possible solution to this problem, but at the cost of much smaller $3 f$ signal amplitude than at the first harmonic, especially for small modulation depths (the $n$th harmonic signal scales with the $n$th power of the modulation depth). This approach was not possible in our case due to the weakness of the signals obtained with low modulation depths. In order to insure that the laser locking point coincides with the centre of the absorption line, we preferred a different approach that relies on the adjustment of the reference phase of the lock-in demodulation in such a way that the IM offset background in the $1 f$ signal is cancelled [24]. Yet, the amplitude of this offset-free signal is reduced in comparison to the signal of maximum amplitude. For the weak line, error-signal modelling demonstrated that the additional implementation of balanced detection [25] strongly reduces the influence of the residual laser IM on the locking point. Implementation of the balanced detection should ideally remove the offset in the $1 f$ signal for any detection phase. Experimentally, a small background offset remains. It likely originates from a small phase shift between the signals of the two detectors and can be cancelled by proper adjustment of the lock-in phase.

WM of the seeder may be considered as inapplicable in view of injection seeding operation. Yet, the important parameter is not the frequency-modulation amplitude of the seeder, but the frequency range covered during the pulse build-up time of the power oscillator. Assuming a conservative (fairly large) build-up time of $10 \mu$ s and a laser frequency sinusoidally modulated at $1 \mathrm{kHz}$ with a peak-to-peak amplitude of $60 \mathrm{MHz}$, the frequency excursion range is at maximum $300 \mathrm{kHz}$. This value represents a tiny portion of the free spectral range of a power oscillator, which is typically in the order of a few hundreds of MHz. Obviously, injection seeding will occur only if the slave laser cavity is in resonance with the seed laser frequency during the build-up time. This condition can be realised with appropriate synchronisation circuitry. High passive stability of the master and slave lasers increases the probability of achieving long-term and stable injection seeding. The stability of the seed laser will increase if a higher modulation amplitude can be used. Injection seeding a power oscillator with a seeder frequency stabilised onto a molecular absorption line applying the WMS technique was already demonstrated [26]. The final choice of the frequency modulation will depend on the pulse power oscillator specifications. Whether the laser will be of high- or low-gain type will strongly influence the pulse build-up time and therefore the injection seeding operation timing. The pulse emission mode of the laser, either single or double, and the pulse repetition rate will also impact on the overall timing. In order to secure further injection seeding, we considered a $60-\mathrm{MHz}$ peak-to-peak frequency-modulation amplitude as the upper acceptable value.

For each of the on-line lasers, a separate absorption cell is used as a frequency reference unit. In order to enable a more precise stabilisation of the lasers, each cell is filled with water vapour under reduced pressure such as to decrease the pressure-broadened line width to the Doppler limit $(\sim 1.2 \mathrm{GHz}$ FWHM $)$ and to increase the peak absorption (see Fig. 1). Optimal cell (water-vapour) pressure lies in the 10-23 mbar range, considering that the absorption coefficient is proportional to the pressure and that the water-vapour saturation pressure at $20^{\circ} \mathrm{C}$ is $23 \mathrm{mbar}$. In order to comply with the above-mentioned requirement of 60-MHz laser frequency modulation amplitude, the modulation index $m$ (defined as the ratio of the laser frequency modulation amplitude to the absorption line width, FWHM) was kept at a low value of

\begin{tabular}{|c|c|c|c|c|c|c|}
\hline $\mathrm{H}_{2} \mathrm{O}$ line & Cell length & Optical path length & $\mathrm{H}_{2} \mathrm{O}$ partial pressure & Total pressure & Cell transmission & Cell $\mathrm{H}_{2} \mathrm{O}$ line absorption \\
\hline $\mathrm{S}$ & $15 \mathrm{~cm}$ & $75 \mathrm{~cm}$ & 22 mbar & 22 mbar & $57 \%$ & $37.5 \%$ \\
\hline M & $18 \mathrm{~cm}$ & $126 \mathrm{~cm}$ & 22 mbar & 22 mbar & $46 \%$ & $7.5 \%$ \\
\hline W & $10 \mathrm{~cm}$ & $400 \mathrm{~cm}$ & 22 mbar & 30 mbar & $35 \%$ & $1.3 \%$ \\
\hline
\end{tabular}

TABLE 3 Characteristics of the water-vapour reference cells implemented in the experimental setup (lines S, M and W). The sixth column represents the overall transmission in each reference cell arrangement (including mirror losses) outside any water-vapour absorption line, whereas the last column represents the contribution of the water-vapour absorption in the centre of each line 


\begin{tabular}{lc}
\hline Line & Frequency stabilisation method \\
\hline Strong & WMS, $1 f$ detection and proper detection phase \\
Medium & WMS, $1 f$ detection and proper detection phase \\
Weak & WMS with balanced detection, $1 f$ detection and proper detection phase \\
Off-line & Offset locking to line $\mathrm{W}$ at $18.8 \mathrm{GHz}$ with a low-pass filter, $1 f$ detection \\
\hline
\end{tabular}

TABLE 4 Summary of the selected locking schemes for the stabilisation of the four lasers $m=0.05$ for a $1.2-\mathrm{GHz}$-wide line. For such a small modulation index, the error-signal amplitude is proportional to $m$ and is strongly reduced compared to the maximum signal obtained for $m \cong 2$ [24]. This signal reduction has to be compensated by sufficiently long optical path lengths in the reference absorption cells, especially for the weak line.

The reference cells used for stabilisation to lines $\mathrm{S}$ and $\mathrm{M}$ consist of a sealed cylindrical glass tube filled with 22 mbar of pure water vapour and are placed in a multi-pass configuration made of two external mirrors. The detailed characteristics of the cells are listed in Table 3. For line W, a commercial Whitestyle multi-pass cell (model 2.4-PA, Infrared Analysis Inc.) is used. This cell was filled in controlled pressure conditions and is closed by two valves. In order to implement balanced detection to remove the large optical offset in the $1 f$ signal, the laser beam is split into two parts before the absorption cell. The first part passes through the cell before being detected, whereas the second is directly detected by a reference photodiode. The two signals are then subtracted. Table 4 summarises the type of laser and the stabilisation scheme used for each wavelength reference. The choice of the type of laser used for each line is partly arbitrary (except for the W line), since the lasers may be interchanged for each line. For line W, an ECDL is preferred since PZT modulation can be used in order to further reduce the residual laser IM.

The implementation of three independent reference cells in the system allows the optimisation of each unit separately with respect to the different absorption strengths of the ref-
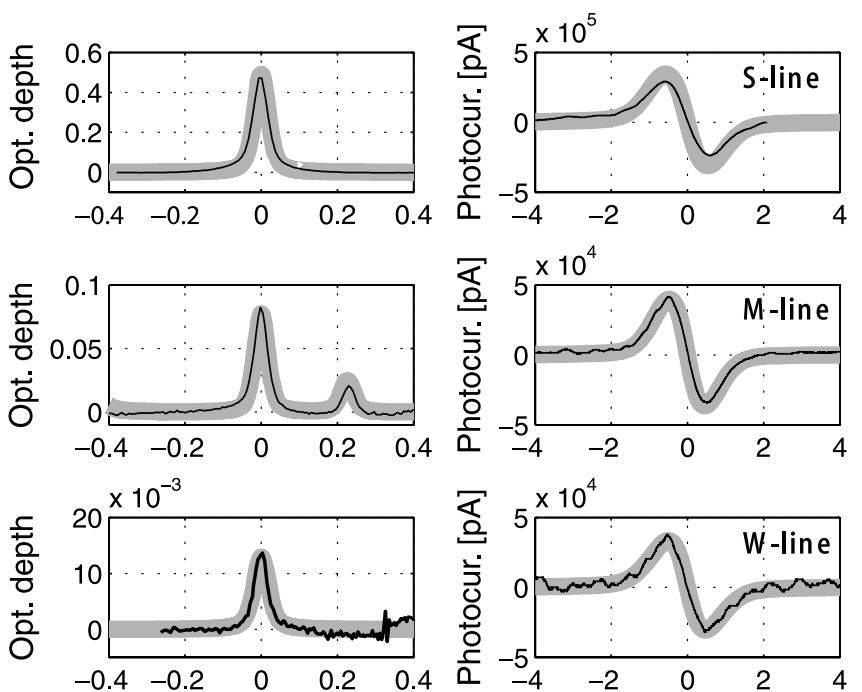

a

Rel. wavenumber [cm-1] b

Relative freq. [GHz]

FIGURE 5 Direct absorption profiles (a) and error signals (b) for the strong $(\mathrm{S})$, medium $(\mathrm{M})$ and weak $(\mathrm{W})$ water-vapour lines obtained with their respective reference absorption cells (cf. Table 3). Simulated (thick grey line) and measured (thin black line) signals are compared. The displayed error signals correspond to a modulation index $m=0.05$ for each line erence lines. This configuration has been preferred to the use of a single absorption cell with either a single detector and a frequency multiplexing of three superimposed laser beams or with three beams spatially separated in the cell and detected with three different photodiodes. Indeed, beam superposition with frequency multiplexing presents some risks for the balanced detection used for the weak line due to a cross-talk sensitivity between the various wavelengths. Coupling all beams into the same cell and separating them at its output is difficult and favours the occurrence of interferences between the beams (mainly in the case of a multi-pass cell).

Absorption lines of about $1.2 \mathrm{GHz}$ FWHM were measured for all water-vapour reference units. Figure 5 illustrates the absorption signal observed for each line and the corresponding absorption calculated for the same conditions using the ESA water-vapour database [17]. The error signal obtained for each $\mathrm{H}_{2} \mathrm{O}$ line in the case of a modulation index $m=0.05$ is also presented in this figure. These signals are in good agreement with the results of simulations performed for each reference unit using a theoretical model that we have developed. Real experimental conditions and laser parameters have been taken into consideration in the calculations. The $1 f$ signals measured for the lines $\mathrm{S}$ and $\mathrm{M}$ show a good signal-to-noise ratio that makes them fully exploitable as an error signal for laser stabilisation. The signal-to-noise ratio is lower for the line $\mathrm{W}$, due to the weaker strength of the absorption coefficient and to the presence of an optical fringe pattern induced by an etalon effect that distorts the signal. Even if we worked with antireflection-coated optical elements, tilted at non-normal incidence and with wedged surfaces in some cases (for example the cell window), a residual etalon effect was nevertheless present and could not be fully suppressed. This residual etalon noise amounts to an equivalent absorption of $6 \times 10^{-4}$ (in terms of optical depth) in the best case, but was up to one order of magnitude higher in the case of unsatisfactory optical alignment. Residual etalon effects have also been observed in some cases for the lines $\mathrm{S}$ and $\mathrm{M}$, but at a much lower level compared to the absorption signal. In that case, the etalon noise was produced by multiple reflections on the windows of the sealed glass-tube reference cells. For the line $\mathrm{W}$, the period of the fringe pattern corresponds to an optical cavity of $20 \mathrm{~cm}$, which is twice the distance between the cell mirrors. The etalon effect results in this case from optical interferences between adjacent focal spots on the mirrors of the multi-pass cell [27], which is known to give rise to interference fringes in multi-pass cells [28].

The frequency-dependent fringe pattern induced by the etalon effect is not stable, but tends to slowly drift over time due to thermal effects. This drift alters the laser locking frequency, since the zero-crossing point of the error signal is slightly shifted when a periodic pattern is superimposed on the absorption-line derivative signal. In consequence, the laser frequency fluctuates and the stability is degraded. 


\section{2}

\section{Off-line locking}

The stabilisation of the off-line laser was achieved by locking it relatively to one of the on-line lasers stabilised to a water-vapour absorption line, at a precise frequency difference. As phase coherence between the two lasers is not necessary in our case, an optical frequency lock loop is sufficient for the offset locking, which strongly relaxes the stringent requirements on the control electronics bandwidth that is needed in frequently used offset-locking techniques based on an optical phase-lock loop [29,30]. The basic principle of an offset-locking scheme consists in controlling the frequency difference between the master (on-line) and slave (offline) lasers, detected as the beat-note signal between the two lasers, using a high-bandwidth photodetector. In our case, the smallest frequency difference from the off-line wavelength is $18.791 \mathrm{GHz}$ from line $\mathrm{W}$, while line $\mathrm{S}$ lies $51.15-\mathrm{GHz}$ away (see Fig. 1 and Table 1). As currently available commercial detectors are limited to $40 \mathrm{GHz}$, the laser locked to the line $\mathrm{W}$ is the best-suited reference and has thus been selected.

Our approach to stabilise the beat frequency and thus to realise the offset locking is based on an adaptation of a modulation-free filter technique [31]. By adding a modulation to one of the lasers, we generated an error signal that is very similar to the signal used for on-line locking. The principle of the implemented offset-locking scheme is sketched in Fig. 6. A fraction of the optical power of the master laser is split off and superimposed with the slave laser on a fast photodetector (New-Focus 1437M, 25-GHz bandwidth). The beat frequency is then converted to low frequency using a doublebalanced mixer with the desired offset frequency applied to the mixer local oscillator port. An 18.791-GHz dielectric resonator oscillator (DRO) is used as a reference signal for this frequency down-conversion. In combination with a low-pass filter, this arrangement may be seen as creating an artificial reference line for the beat frequency, centred at the frequency of the reference oscillator. After amplification, the power of the radio-frequency signal is measured using an envelope detector. When the wavelength of one of the lasers is modulated, derivatives of the filter transfer function are obtained at the harmonics of the modulation frequency in the same way as in WMS for a molecular absorption line. The first-harmonic signal is used as an error signal to control the slave laser frequency. The use of a narrow filter with steep slope enables efficient stabilisation of the beat frequency at the requested offset frequency.

To illustrate the operation of the offset-locking scheme, the evolution of the electrical signal through the various com-

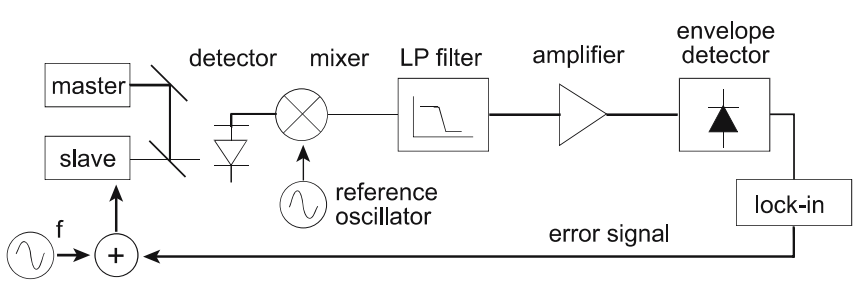

FIGURE 6 Principle of the offset-locking scheme based on the stabilisation of the beat signal using a low-pass (LP) filter and wavelength modulation of the slave laser ponents, from the detected beat signal to the generation of the error signal, is shown in Fig. 7. The beat signal measured on the photodetector has been observed by changing the emission frequency of the slave laser over $\pm 6 \mathrm{GHz}$ around $18.8 \mathrm{GHz}$ with respect to the master laser. The small variation of the beat-signal amplitude at the detector output (Fig. 7a) is due to the change of the master laser emission power, to the slightly frequency-dependent response of the detector and to minor misalignments of the two beams on the detector. A typical beat-signal amplitude of $1 \mathrm{mV}$ is obtained for an incident optical power of around $1 \mathrm{~mW}$ for each laser. The filtering effect of the low-pass filter on the down-converted beat signal at the filter output is clearly visible (Fig. 7b). When the beat signal is continuously scanned over $\pm 1 \mathrm{GHz}$ around its central value, the offset-locking output directly displays the filter frequency response (Fig. 7c), showing the strong resemblance with an absorption line. To be precise, the filter rather acts as a narrow transmission feature (attenuation occurs outside the filter spectral width, whereas it takes place inside an absorption line). However, the effects of these two types of function on the $1 f$ error signal are totally equivalent. A 3-dB filter cut-off frequency of around $230 \mathrm{MHz}$ was measured, yielding a corresponding microwave feature of $460-\mathrm{MHz}$ FWHM, compared to the 1.2-GHz FWHM of the water absorption lines. This artificial absorption line presents a slightly asymmetric shape, which results from the variation of the amplitude of the beat signal induced by the current ramp applied to the slave laser (DFB laser) in order to scan the beat signal. The narrow dip in the centre of the line is induced by the envelope detector, which has a low-frequency cut-off at $10 \mathrm{MHz}$. This dip does not affect the $1 f$ signal (Fig. 7d) measured with lock-in detection for modulation indices $m$ as low as 0.1 (defined with respect to the filter FWHM of $460 \mathrm{MHz}$, thus corresponding to a modulation amplitude of $45 \mathrm{MHz}$ ), as its effect is smoothed by the modulation.

Because the beat-note frequency is the same for the slave laser locked to the higher or lower absolute frequency than the master laser, a two-fold degeneracy occurs in this scheme.
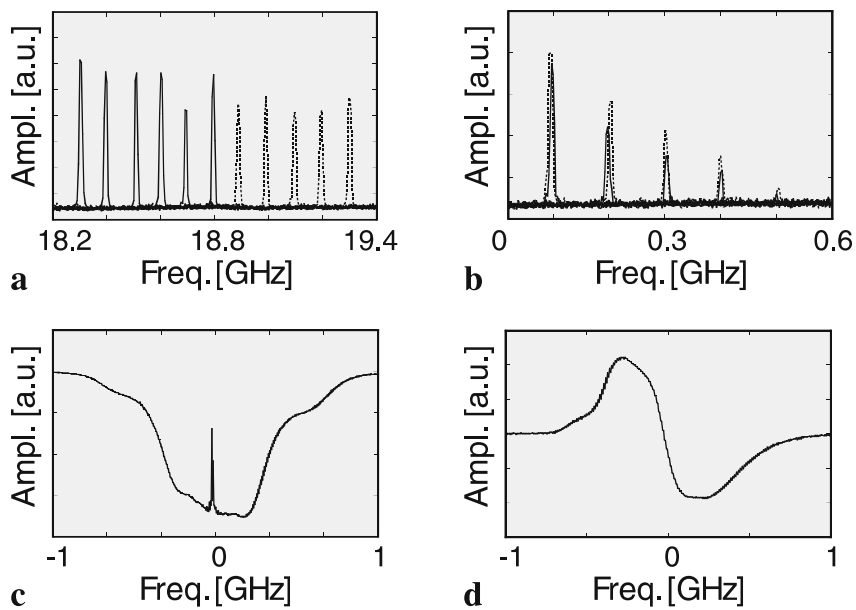

FIGURE 7 Signal evolution at different stages of the offset-locking setup for a beat node around $18.8 \mathrm{GHz}$; (a) fast detector output; (b) low-pass-filter output; (c) frequency response after the envelope detector at the output of the setup for a continuous wavelength scan applied to the slave laser; (d) derivative signal (first harmonic) of the filter function obtained using lock-in detection 
However, the two corresponding frequencies are far away from each other - twice the oscillator reference frequency, i.e. $37.6 \mathrm{GHz}$ for our concern - so this does not really cause a problem. A band-pass filter could also be used in the developed offset-locking technique, but an additional two-fold degeneracy appears in such a configuration, separated by twice the filter centre frequency. These two points can be quite close (e.g. a few $\mathrm{GHz}$ apart), which can lead to an incorrect locking point. Another advantage offered by the use of a lowpass filter is an improved thermal stability: whereas the centre frequency of a band-pass filter can slightly drift with temperature, it remains zero for a low-pass filter.

The modulation of the beat-note frequency that is required in the offset-locking scheme can in principle be accomplished by WM of either the master or the slave laser. For example, the WM already applied to the master laser for direct locking to the $\mathrm{W}$ line could also serve to generate the offset-locking error signal. However, in order to enable an independent adjustment of the modulation index for each error signal (direct and offset locking), modulation of the slave laser has been chosen. In addition, different modulation frequencies have been chosen to avoid risk of cross talk between the master and slave laser modulations. Hence, the master ECDL is PZT modulated at $1 \mathrm{kHz}$, while $50-\mathrm{kHz}$ injection current modulation is used for the slave laser (DFB laser).

For the off-line laser, larger modulation indices may be used compared to the on-line lasers for two reasons. First, as the width of the offset-locking filter function is approximately three times narrower than the width of the considered watervapour absorption lines, a three times larger modulation index ( $m \approx 0.13$ ) may be used, keeping the laser spectrum broadening within $60 \mathrm{MHz}$ (the modulation index is always referred to the width of the spectral feature of interest). Furthermore, as the slave laser is locked off-line on a broad $(7 \mathrm{GHz})$ flat background, its stability requirements are less stringent than for other lasers. A larger modulation depth can therefore be used without affecting the overall performances of the DIAL, providing that the resonance criterion for injection seeding is respected. A peak-to-peak modulation amplitude of $200 \mathrm{MHz}$, corresponding to a modulation index $m \approx 0.45$, seems acceptable in this context.

A critical parameter for the performance of the offsetlocking scheme is the accuracy and the precision of the DRO. Its absolute frequency can be slightly adjusted $( \pm 10 \mathrm{MHz})$ and was checked with an electrical spectrum analyser. A 90-h stability measurement showed that after an initial frequency drift of $400 \mathrm{kHz}$ during a 24-h warming-up time, the DRO was stable within $30 \mathrm{kHz}$ over the following $65 \mathrm{~h}$. Even with warm-up, these drifts are orders of magnitude smaller than the target stability of the off-line-locked laser.

The $1 f$ signal is free from background offset due to IM for any detection phase of the lock-in demodulation. Therefore, the offset-locking scheme behaves like a molecular absorption line probed by pure WM. This property is favourable, as the detection phase may be adjusted in order to maximise the amplitude of the $1 f$ signal and not so as to cancel the offset level.

The amplitude of the derivative signal also shows a good linearity with the optical power generating the beat signal (the square root of the product of the optical power from the two lasers). Some small distortions occur in the signal when the optical power is reduced, but they do not prevent laser locking. The error signal could be detected with a good signal-to-noise ratio for optical powers as low as $30 \mu \mathrm{W}$ from each laser.

\section{$5 \quad$ Stabilised laser performances}

The frequency stability of the multi-wavelength reference system was evaluated using a high-precision wavemeter (HighFinesse WS/Ultimate, $10-\mathrm{MHz}$ accuracy). To correctly interpret the stability of the lasers, the stability of the wavemeter itself was determined with a reference laser stabilised to a $\mathrm{Rb}$ Doppler-free saturated absorption resonance at $780 \mathrm{~nm}$. This $\mathrm{Rb}$ laser was stable to $2 \times 10^{-12}$ (i.e. less than $1 \mathrm{kHz}$ ) over integration times between $1 \mathrm{~s}$ and 1 day [18]. The wavemeter output was recorded with an integration time of $1 \mathrm{~s}$ during $90 \mathrm{~h}$. The results are reported in Fig. 8 in terms of the Allan deviation. The wavemeter stability is about $2 \mathrm{MHz}$ at $\tau=1$-s integration time and around $10 \mathrm{MHz}$ over 1 day.

The stabilities of the lasers locked to their respective water-vapour absorption lines are reported in Fig. 8 as well. The laser stabilities amount to $1-10 \mathrm{MHz}$ at 1 to $10 \mathrm{~s}$ and less than $10 \mathrm{MHz}$ at $1000 \mathrm{~s}$, while extrapolated stabilities over one day lie between $15 \mathrm{MHz}$ and $100 \mathrm{MHz}$, depending on the absorption strength of the reference line. The first laser locked to line $\mathrm{S}$ was PZT modulated at $1 \mathrm{kHz}$ with a modulation index of 0.03 (with respect to the $\sim 1.2 \mathrm{GHz}$ width of the water-vapour line), which broadened its spectrum to $40 \mathrm{MHz}$. The observed frequency stability of this laser is clearly limited by the stability of the wavemeter over all averaging times, but is below $10 \mathrm{MHz}$. The slight differences between the two curves can be explained by the different wavelengths at which the stabilities were measured and by the slightly different instrument settings (short-term influence only). Based on the system parameters and taking into account etalon interference fringes with a maximum amplitude equivalent to an absorption assessed to $6 \times 10^{-4}$, we estimated a long-term stability better than $0.5 \mathrm{MHz}$. Typical laser emitter performances for DIAL water-vapour applications require a frequency stability better than $210 \mathrm{MHz}(1 \sigma)$ [32] for the on-line wavelength. For a space DIAL [33], the drift over several months cannot ex-

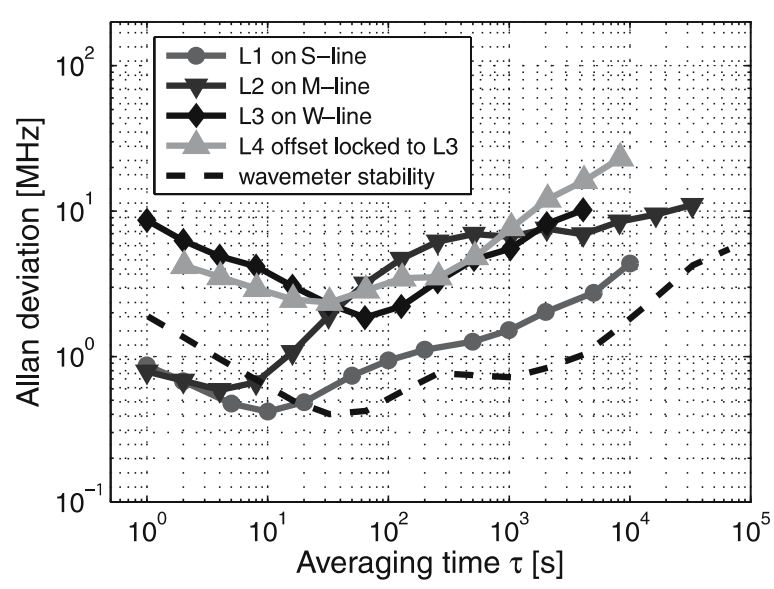

FIGURE 8 Stability of the lasers locked to their corresponding absorption lines. The stability of the wavemeter used for the characterisation of the lasers has been measured at $780 \mathrm{~nm}$ 


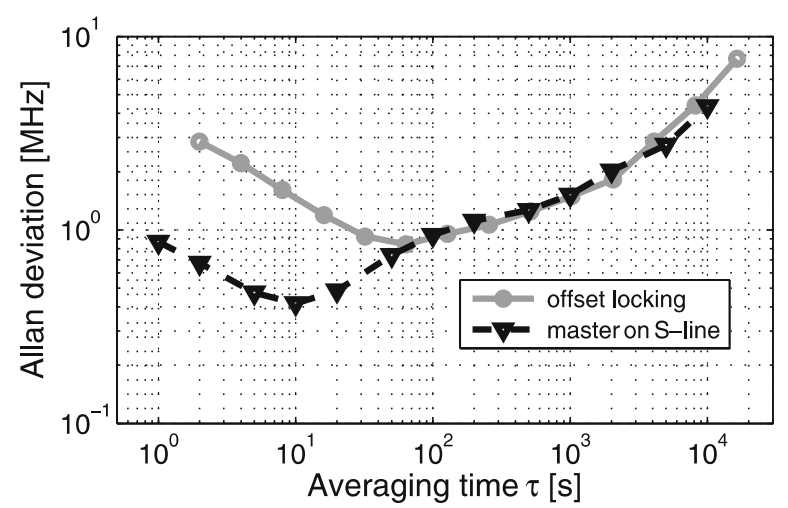

FIGURE 9 Evaluation of the offset-locking stability in terms of the Allan deviation of the frequency difference between the master and slave lasers. It represents a measurement of the slave laser stability (offset-locked) relatively to the master laser. The master laser was locked to line $\mathrm{T}_{1}$ (line strength equivalent to the $\mathrm{S}$ line) and the slave laser was offset-locked $10 \mathrm{GHz}$ away using the offset-locking scheme with a $10-\mathrm{GHz}$ reference frequency from a frequency generator

ceed $60 \mathrm{MHz}$. Therefore, we considered a value of $60 \mathrm{MHz}$ as the reference target on a 24-h basis for the lasers. The laser locked to the $\mathrm{S}$ line largely fulfils this limit.

For the second laser locked to line M, the 'bump' observed around $300 \mathrm{~s}$ is due to some instabilities arising from etalon fringe effects in the (home-made) multi-pass reference cell. The first laser also demonstrated a similar feature, but much less pronounced, as the strength of the $\mathrm{M}$ line is lower than the $\mathrm{S}$ line by approximately one order of magnitude. The time scale of these instabilities corresponds to the switching of the air conditioning in the laboratory. In the presence of an etalon noise with an assessed amplitude equivalent to an absorption of $6 \times 10^{-4}$, the stability of this laser was estimated to be $2 \mathrm{MHz}$, a value slightly lower than the observations. The plateau around $8 \mathrm{MHz}$ for times between $500 \mathrm{~s}$ and $4000 \mathrm{~s}$ likely represents the actual long-term stability for this laser, while this behaviour is hidden by the increasing drift of the wavemeter at longer term. This laser was modulated at $50 \mathrm{kHz}$ by current injection with a modulation index of 0.04 , which corresponds to a $50-\mathrm{MHz}$ broadened laser spectrum.

When extrapolated to $24 \mathrm{~h}$, the stability of the third laser locked to line $\mathrm{W}$ reaches $50 \mathrm{MHz}$. It shows the largest frequency drift over several hours due to the relatively larger influence of the etalon noise on the weak absorption line signal. This drift critically depends on the collimation of the laser beam and on the alignment of the multi-pass cell. For interference fringes with an assessed amplitude equivalent to an absorption of $2 \times 10^{-3}$, we estimated a stability limit of $50 \mathrm{MHz}$ for this laser, in the range of the observations.

For the stability measurement of the fourth laser reported in Fig. 7, the off-line laser was offset locked to the third laser stabilised to the $\mathrm{W}$ line. The achieved stability is about $100 \mathrm{MHz}$ on an extrapolated daily basis, whereas a value similar to the master laser was expected. The observed difference results from the fact that the stability measurements of these two lasers were not realised at the same time. The difference in the mid- and long-term behaviour can be explained by a lower stability of the master laser during the evaluation of the offsetlocked laser, caused by a less optimised optical adjustment of the master laser beam within the White-type multi-pass cell.
Indeed, it will be demonstrated below that the offset-locked slave laser stability equals that of the master laser. Thus, the reduced stability of the off-line laser observed in Fig. 7 is a consequence of the susceptibility of the master laser to the optical adjustment.

In order to demonstrate the correct operation of the offsetlocking setup, two close strong absorption lines separated by $9.5 \mathrm{GHz}$ were considered around $935.75 \mathrm{~nm}$ for locked laser frequency stability measurement. These lines are marked as $\mathrm{T}_{1}$ and $\mathrm{T}_{2}$ in Fig. 1. The master laser (laser 3) was locked to line $\mathrm{T}_{1}$ using the 4-m multi-pass absorption cell, while the other line, $\mathrm{T}_{2}$, served as a frequency discriminator for the slave laser (laser 4) offset locked $10 \mathrm{GHz}$ away from laser 3 - an additional shift of $0.5 \mathrm{GHz}$ was added to set laser 4 on the slope of the absorption line. The slave laser was current modulated and an external frequency generator replaced the $18.791-\mathrm{GHz}$ reference oscillator (DRO). The wavelength of laser 3 was additionally monitored by the wavemeter.

The stability of the frequency difference between the offset-locked slave laser and the master laser is shown in Fig. 9. It is compared to the stability of laser 1 when locked to the $\mathrm{S}$ line (cf. Fig. 8). At short-term, the difference in the observed stabilities can be explained by the different modulation amplitudes applied to the lasers. Starting from an averaging time above $1 \mathrm{~min}$, the stabilities are identical, indicating that the offset-locked laser follows the master laser. Possible instabilities provoked by the offset locking are masked by the drift of the measurement setup, which is dominated by the wavemeter drift.

\section{6}

\section{Conclusions and perspectives}

A four-wavelength optical reference system for water-vapour DIAL applications in the 935-nm range has been developed. Three lasers are locked to water-vapour absorption lines while the fourth laser is locked off-line. Laser locking is realised based on the WMS technique and firstharmonic detection at a proper detection phase to cancel the residual IM contribution. Low modulation indices are used to maintain the modulation-broadened laser spectrum below $60 \mathrm{MHz}$. Two types of semiconductor laser sources were implemented in the setup, home-made ECDLs and DFB lasers.

For lasers locked to water-vapour lines of strengths larger than $5 \times 10^{-23} \mathrm{~cm}^{-1} /\left(\mathrm{mol} \mathrm{cm}^{-2}\right)-$ strong and medium lines in this work - frequency stabilities below $15 \mathrm{MHz}$ were observed over one day. The observations were limited by the precision of the measurement instrument. Using a long optical path length reference cell as a frequency discriminator would allow more precise measurements of the laser stability. For a laser locked to a weak absorption line with a strength around $\left.4 \times 10^{-24} \mathrm{~cm}^{-1} /(\mathrm{mol} \mathrm{cm})^{-2}\right)$, the frequency stability degrades to $50 \mathrm{MHz}$ (over $24 \mathrm{~h}$ ) or worse, depending on the optical alignment and resulting optical interference fringes. This situation is not satisfactory in view of applications requesting a long-term stability better than $60 \mathrm{MHz}$ over many days, like a space DIAL.

As long as a proper stabilisation scheme for injection seeding allows it, increasing the modulation index (i.e. the frequency-modulation amplitude) is a first possibility to enlarge the signal-to-noise ratio in the error signal and, in con- 
sequence, to improve the laser stability. A possible solution to reduce the instabilities arising from etalon fringes is to mechanically modulate the fringe spacing, so that the integrated photocurrent is averaged to zero [34]. Another approach consists in applying an additional frequency jitter to the laser in order to reject the fringe signal $[27,35]$. Making use of a longer optical path length is a third possibility to increase the signal-to-noise ratio of a weak absorption signal. Increasing the optical path length from $4 \mathrm{~m}$ to $25 \mathrm{~m}$ would generate an absorption of $7.8 \%$, comparable to the value that we used for the M line (7.5\%). Larger multi-pass cells can be found; however, their size and mass will increase as well. A convenient solution to achieve a longer path length would be the use of a hollow-core photonic crystal fibre (HC-PCF) filled with water vapour at reduced pressure as a reference cell. Locking a near-infrared laser to an acetylene-filled HC-PCF has been demonstrated in an all-fibre configuration [36] and first tests we made with a HC-PCF filled with carbon dioxide at $1.57 \mu \mathrm{m}$ are quite promising. Additional advantages offered by a HC-PCF-based cell are in terms of volume, mass and mechanical stability.

The offset-locking scheme we have reported in this paper is revealed to be quite robust and simple. The stability of the offset slave laser is limited by the stability of the master laser, at least in the MHz-precision range. This principle can be applied in any wavelength region and the frequency offset can be easily varied in the range $0-25 \mathrm{GHz}$ by changing the frequency of the reference local oscillator. The major limitation of this technique lies in the maximum achievable offset frequency, which is limited by the bandwidth of the fast photodetector ( $25 \mathrm{GHz}$ in the present configuration, up to $45 \mathrm{GHz}$ with a commercial fibre-pigtailed detector). However, much larger offset frequencies can be obtained by combining the proposed technique with an optical frequency comb (OFC). An OFC made of tens to hundreds of equally spaced optical frequencies with several gigahertz frequency spacing can be generated from a single-mode laser [37]. By locking one line of the comb to a molecular absorption line, all the sidebands of the OFC have a determined and stable frequency. One of these lines may then be used as a master laser to offset lock a slave laser some gigahertz away. With such a configuration, offset locking of several tens or hundreds of gigahertz can be achieved using standard electronics and photodetectors with a limited bandwidth of a few gigahertz only. Recently, we achieved very promising results using an all-fibre OFC configuration in the $1.55-\mu \mathrm{m}$ region, with an offset locking larger than $320 \mathrm{GHz}$, limited only by the spectral tuning range of the slave laser. A single comb could even be used to lock several lasers on a master laser, for instance the off-line, weak-line or even medium-line lasers. Employing a stabilised (self-referenced) mode-locked femtosecond laser and a suitable accurate local oscillator would even avoid the necessity of any water-vapour reference [38].

ACKNOWLEDGEMENTS The authors would like to acknowledge the European Space Agency ESA (ESTEC Contract No. $17267 / 03 / \mathrm{NL} / \mathrm{CH})$, the Canton de Neuchâtel, the Ecole Polytechnique Fédérale de Lausanne and the Swiss National Science Foundation (Projects
2100-066821.01 and R'Equip 2160-06749) for their financial support. The authors wish to thank Robert Drullinger for reading the manuscript, Enzo Nava (CESI-Milano) for helpful discussions about injection seeding and power oscillators and Valentin Mitev for useful communications on atmospheric measurement techniques.

\section{REFERENCES}

1 T. Weckwerth, V. Wulfmeyer, R. Wakimoto, R. Hardesty, J. Wilson, R. Banta, Bull. Am. Meteorol. Soc. 80, 2339 (1999)

2 R. Schotland, in Proc. Fourth Symp. Remote Sensing of the Environment (Environmental Research Institute of Michigan, Ann Arbor, MI, 1966), pp. 271-273

3 S. Ismail, E. Browell, Appl. Opt. 28, 3603 (1989)

4 J. Bösenberg, Appl. Opt. 37, 3845 (1998)

5 V. Wulfmeyer, J. Bösenberg, Appl. Opt. 37, 3825 (1998)

6 K. Ertel, Application and Development of Water Vapor DIAL Systems, Dissertation, Fachbereich Geowissenschaften der Universität Hamburg (2004)

7 E. Browell, S. Ismail, W. Hall, A. Moore, S. Kooi, V. Brackett, M. Clayton, J. Barrick, F. Schmidlin, N. Higdon, S. Melfi, D. Whiteman in Advances in Atmospheric Remote Sensing with Lidar, Selected Papers 18th Int. Laser Radar Conf. (ILRC), Berlin, 22-26 July 1996, ed. by A. Ansmann, R. Neuber, P. Rairoux, U. Wandinger (Springer, Berlin, 1997), pp. 289-295

8 G. Ehret, K. Hoinka, J. Stein, A. Fix, C. Kiemle, G. Poberaj, J. Geophys. Res. 104, 31351 (1999)

9 G. Poberaj, A. Fix, A. Assion, M. Wirth, C. Kiemle, G. Ehret, Appl. Phys. B 75, 165 (2002)

10 D. Bruneau, P. Quaglia, C. Flamant, M. Meissonnier, J. Pelon, Appl. Opt. 40, $3450(2001)$

11 E. Browell, S. Ismail, W. Grant, Appl. Phys. B 67, 399 (1998)

12 E. Gérard, D. Tan, L. Garand, V. Wulfmeyer, G. Ehret, P. Di Girolamo, Bull. Am. Meteorol. Soc. 85, 237 (2004)

13 V. Wulfmeyer, C. Walther, Appl. Opt. 40, 5304 (2001)

14 J. Barnes, N. Barnes, L. Wang, W. Edwards, IEEE J. Quantum Electron. QE-29, 2684 (1993)

15 K. Ertel, H. Linne, J. Bösenberg, Appl. Opt. 44, 5120 (2005)

16 A. Fix, V. Weiss, G. Ehret, Pure Appl. Opt. 7, 837 (1998)

17 R. Schermaul, R. Learner, D. Newnham, J. Ballard, N. Zobov, D. Belmiloud, J. Tennyson, J. Mol. Spectrosc. 208, 43 (2001)

18 C. Affolderbach, G. Mileti, Rev. Sci. Instrum. 76, 073108 (2005)

19 J. Supplee, E. Whittaker, W. Lenth, Appl. Opt. 33, 6294 (1994)

20 N. Goldstein, S. Adler-Golden, Appl. Opt. 32, 5849 (1993)

21 O. Grohe, H. Gottschling, H. Jennewein, T. Tschudi, Opt. Eng. 40, 529 (2001)

22 C. Fitzgerald, G. Koch, A. Bullock, A. Dharamsi, Proc. SPIE 3945, 98 (2000)

23 G. Koch, A. Cook, C. Fitzgerald, A. Dharamsi, Opt. Eng. 40, 525 (2001)

24 S. Schilt, L. Thévenaz, P. Robert, Appl. Opt. 42, 6728 (2003)

25 V. Pevtschin, S. Ezekiel, Opt. Lett. 12, 172 (1987)

26 G. Koch, M. Petros, J. Yu, U. Singh, Appl. Opt. 41, 1718 (2002)

27 D. Cassidy, J. Reid, Appl. Phys. B 29, 279 (1982)

28 G. Gagliardi, L. Gianfrani, Opt. Laser. Eng. 37, 509 (2002)

29 U. Gliese, T. Nielsen, M. Bruun, E. Lintz Christensen, K. Stubkjaer, S. Lindgren, B. Broberg, IEEE Photon. Technol. Lett. 4, 936 (1992)

30 R. Ramos, P. Gallion, D. Erasme, A. Seeds, A. Bordonalli, Opt. Lett. 19 , 4 (1994)

31 G. Ritt, G. Cennini, C. Geckeler, M. Weitz, Appl. Phys. B 79, 363 (2005)

32 J. Bösenberg, in Lidar, Range-Resolved Optical Remote Sensing of the Atmosphere, ed. by C. Weitkamp (Springer, Berlin Heidelberg, 2005), p. 213

33 A. Hélière, E. Armandillo, Y. Durand, A. Culoma, R. Meynart, in Proc. Fifth Int. Conf. Space Optics, paper 1, session 1, Toulouse, 30 March2 April 2004 [ESA SP-554 2004]

34 J. Silver, A. Stanton, Appl. Opt. 27, 1914 (1988)

35 C. Sun, E. Whittaker, Appl. Opt. 31, 4998 (1992)

36 F. Benabid, F. Couny, J. Knight, T. Birks, P. Russell, Nature 434, 488 (2005)

37 K.-P. Ho, J. Kahn, IEEE Photon. Technol. Lett. 5, 721 (1993)

38 L. Hollberg, S. Diddams, A. Bartels, T. Fortier, K. Kim, Metrologia 42, S105 (2005) 